\title{
Avoiding Arterial Hypotension in Preterm Neonates (AHIP) - A Single Center Randomised Controlled Study Investigating Simultaneous Near Infrared Spectroscopy Measurements of Cerebral and Peripheral Regional Tissue Oxygenation and Dedicated Interventions
}

OPEN ACCESS

Edited by:

Eugene Dempsey,

University College Cork, Ireland

Reviewed by:

Kai König,

Mercy Hospital for Women, Australia

*Correspondence:

Gerhard Pichler gerhard.pichler@medunigraz.at

Specialty section: This article was submitted to Neonatology,

a section of the journal

Frontiers in Pediatrics

Received: 22 October 2017 Accepted: 16 January 2018 Published: 01 February 2018

Citation: Pichler G, Höller N, Baik-Schneditz N, Schwaberger B, Mileder L, Stadler J, Avian A, Pansy $J$ and Urlesberger $B$ (2018) Avoiding Arterial Hypotension in Preterm Neonates (AHIP) A Single Center Randomised Controlled Study Investigating

Simultaneous Near Infrared Spectroscopy Measurements of Cerebral and Peripheral Regional Tissue Oxygenation and Dedicated Interventions.

Front. Pediatr. 6:15 doi: 10.3389/fped.2018.00015
Gerhard Pichler,2*, Nina Höller 1,2, Nariae Baik-Schneditz,2, Bernhard Schwaberger ${ }^{1,2}$, Lukas Mileder ${ }^{1,2}$, Jasmin Stadler, ${ }^{1,2}$, Alexander Avian ${ }^{3}$, Jasmin Pansy ${ }^{1,2}$ and Berndt Urlesberger ${ }^{1,2}$

${ }^{1}$ Research Unit for Neonatal Micro- and Macrocirculation, Department of Pediatrics and Adolescent Medicine, Medical University of Graz, Graz, Austria, ${ }^{2}$ Division of Neonatology, Department of Pediatrics and Adolescent Medicine, Medical University of Graz, Graz, Austria, ${ }^{3}$ Institute for Medical Informatics, Statistics and Documentation, Medical University of Graz, Graz, Austria

Introduction: Up to $50 \%$ of preterm infants admitted to intensive care units require cardiocirculatory support. The aim of the present study was to assess whether simultaneous monitoring of cerebral tissue oxygenation index (cTOI) and peripheral tissue oxygenation index (pTOI) using near-infrared spectroscopy (NIRS) in combination with dedicated intervention guidelines may help avoiding arterial hypotension and catecholamine administration in preterm neonates.

Study design: Preterm neonates $<37$ weeks of gestation were included in a single center randomized controlled study. Blood pressure was measured non-invasively or invasively. In the NIRS group, simultaneous cTOI and pTOI monitoring was used starting within $6 \mathrm{~h}$ after birth for $24 \mathrm{~h}$ to calculate changes in cTOI/pTOI ratio over time. Depending on these changes, interventions including echocardiography, administration of volume or patent ductus arteriosus treatment were performed. In the control group, only routine monitoring and treatment were performed and NIRS signals were not visible. The primary outcome was burden of hypotension within $48 \mathrm{~h}$ after initiation of NIRS monitoring.

Results: 49 preterm neonates were included in each group: NIRS group 33.1 (32.034.0) (median: 25-75 centile) weeks of gestation and control group 33.4 (32.3-34.3) weeks of gestation. In the NIRS group, echocardiography was performed in 17 preterm neonates due to NIRS measurements, whereby six neonates received further treatment. 
Percentage of neonates with any hypotensive episode during the 48-h observational period was $32.6 \%$ in the NIRS group and $44.9 \%$ in the control group $(p=0.214)$. Burden of hypotension (i.e., \%mmHg of mean arterial pressure $<$ gestational age) was $0.0(0.0-2.1) \mathrm{mmHg} h$ in the NIRS group and 0.4 (0.0-3.3) $\mathrm{mmHg} h$ in the control group $(p=0.313)$, with observed burden of hypotension being low in both groups. No severe adverse reactions were observed.

Conclusion: In preterm neonates using simultaneous peripheral and cerebral NIRS measurements for early detection of centralization followed by predefined interventions led to a non-significant reduction in burden of arterial hypotension.

Clinical Trial Registration: www.ClinicalTrials.gov, identifier: NCT01910467.

Keywords: near-infrared spectroscopy, cerebral oxygenation, peripheral-muscle oxygenation, centralization of circulation, early intervention

\section{INTRODUCTION}

Arterial hypotension, irrespective of definition, occurs in up to $20 \%$ of preterm infants, most commonly during the first $48 \mathrm{~h}$ after birth (1). The management of hypotension in preterm infants includes volume administration, inotropes and corticosteroids in refractory cases. Very preterm infants in intensive care units receive fluid boluses in up to $50 \%$, and/or pressor/inotrope medications for cardiovascular support, whereby the most frequent indication for intervention is a low systemic blood pressure in the first few days of life (1-4). Associations between systemic hypotension and fluctuations of blood pressure with cerebral injury such as intraventricular hemorrhage (IVH) $(5,6)$ and periventricular leukomalacia (PVL) $(7,8)$ have been described.

In early stages of shock, neonates often compensate for cardiovascular dysfunction and maintain normal blood pressure. The presence of hypotension is often a late finding $(9,10)$. Early cardiovascular and circulatory signs of inflammation especially in case of sepsis are difficult to interpret in neonates. In sepsis, microvascular dysfunction occurs secondary to perfusion heterogeneity, arteriovenous shunting and impaired autoregulation $(11,12)$. A correlation between microcirculatory abnormalities and organ dysfunction has already been shown (13). Conventional parameters of oxygenation and hemodynamic status may fail to detect microcirculatory dysfunction (14).

Near-infrared spectroscopy (NIRS) enables non-invasive measurement of oxygenation in regions of interest, e.g., cerebral, renal, and in peripheral muscle tissue (15). Most clinical work on cerebral oxygenation especially in term and preterm neonates has been undertaken using "continuous wave spatially resolved technique" NIRS (15-17).

Several studies have already investigated cerebral NIRS and cardiocirculation. Suresh et al. (18) found that cerebral fractional

Abbreviations: TOI, tissue oxygenation index; cTOI, cerebral tissue oxygenation index; pTOI, peripheral tissue oxygenation index; CRP, C-reactive protein; IVH, intraventricular hemorrhage; SVC, superior vena cava; SD, standard deviation; $\mathrm{IQR}$, interquartile range; $\mathrm{HR}$, heart rate; MABP, mean arterial blood pressure; NIRS, near-infrared spectroscopy; $\mathrm{PDA}$, patent ductus arteriosus; $\mathrm{SpO}_{2}$, arterial oxygen saturation; TRDN, transitory respiratory distress of the newborn. oxygen extraction changed at mean arterial blood pressure (MABP) levels below $23 \mathrm{mmHg}$, concluding that cerebral perfusion is probably maintained at MABP levels above $23 \mathrm{mmHg}$. In piglets, cerebral perfusion pressure increased immediately on norepinephrine, whereas cerebral oxygenation as reflected by tissue oxygenation index (TOI) did not improve, except by retransfusion (19).

In a recent study, we described associations between peripheral muscle NIRS parameters, MABP and heart rate (HR) in term and preterm neonates (20). Furthermore, in neonates with inflammatory processes and C-reactive protein (CRP) elevation NIRS measurements showed impaired peripheral oxygenation and perfusion when routine hemodynamic variables were still normal (9).

The primary aim of this study was to examine, whether it is possible to reduce arterial hypotensive episodes (MABP $<$ gestational age, presented in $\% \mathrm{mmHg} / \mathrm{h}$ within the first $48 \mathrm{~h}$ after initiation of NIRS monitoring) and use of inotropes by applying simultaneous cerebral and peripheral muscle NIRS trend monitoring in combination with dedicated early clinical interventions. The secondary aim was to explore potential impact of these interventions on arterial hypotension, the use of inotropes as well as on cerebral injury and mortality.

We hypothesized that by using predefined clinical interventions in case of increase in cerebral/peripheral tissue oxygenation ratio (cTOI/pTOI), hypotensive episodes, and the use of inotropes could be reduced, which may result in less cerebral injury and mortality.

\section{METHODS}

This single center randomized controlled study (AHIP) was carried out at the Division of Neonatology, Medical University of Graz, Austria (EK-Nr: 25-237 ex 12/13; http://ClinicalTrials. gov Identifier: NCT01910467). Preterm neonates $(<37+0$ weeks of gestation) born after premature rupture of membranes, with amnion infection of the mother or increased maternal markers of systemic inflammation (CRP/leukocytes) were included. Written parental consent was obtained for all infants. Further inclusion 
criteria were decision to conduct full life support, age below $6 \mathrm{~h}$ after birth and no use of catecholamines before initiation of NIRS measurements. Exclusion criteria were any congenital malformations.

\section{SAMPLE SIZE}

For this randomized phase I/II study, the sample size was calculated at 49 neonates in each group resulting in a total number of 98 patients. Calculations were based on observations during the first $24 \mathrm{~h}$ after birth in 54 neonates. 15 of these neonates $(27.8 \%)$ had episodes of hypotension (unpublished data). In the present study, participants were randomized into either the NIRS group or the control group. The ratio of allocation was $1: 1$. In case of multiple births, only the first infant was randomized.

\section{INTERVENTIONS}

After study inclusion, NIRS measurements with NIRO 200NX (Hamamatsu Photonics, Hamamatsu City, Japan) were performed in combination with routine monitoring (pulse oximetry, electrocardiography). Blood pressure was measured invasively, when an intra-arterial line was placed routinely and non-invasively with a pneumatic cuff placed around the left upper arm measuring at least every $30 \mathrm{~min}$.

Invasive and nonivasive blood pressure measurements were performed with the IntelliVue MP50 monitor (Philips, Netherland). Data were stored in a polygraphic system (alphatrace digitalMM, B.E.S.T. Medical Systems, Vienna, Austria) for further analysis. Patients in the NIRS and the control group had a cerebral NIRS oximeter probe placed on the left frontal head and a peripheral muscle NIRS oximeter probe placed on the right forearm within $6 \mathrm{~h}$ after birth. The NIRS sensors were repositioned after every $6 \mathrm{~h}$. Interoptode distance was $4.0 \mathrm{~cm}$ for the cerebral optodes and either $3.0 \mathrm{~cm}$ in neonates $>1,500 \mathrm{~g}$ or $2.0 \mathrm{~cm}$ in neonates $<1,500 \mathrm{~g}$ for the peripheral muscle optodes.

Cerebral and peripheral tissue oxygenation (TOI) were measured continuously with a sampling rate of $2 / \mathrm{s}$. The mean of cerebral TOI (cTOI) and peripheral muscle TOI (pTOI) were calculated every hour. After every hour changes of cTOI/pTOI ratio were calculated as a trend monitoring. An increase of cTOI/ pTOI ratio of more than $5 \%$ within a 6 -h interval was assumed to be out of range $(18,20-22)$ and a first sign of centralization (beginning shock).

In the control group, NIRS parameters were not visible and the infants were treated according to routine ("treatment as usual"). In the NIRS group, NIRS parameters were visible and neonates were treated according to predefined intervention guidelines.

According to these guidelines, an echocardiography was indicated, if cTOI/pTOI ratio increased $>5 \%$ within less than $6 \mathrm{~h}$. Echocardiography was performed by neonatologists, who had been trained in neonatal echocardiography at the Division of Cardiology, Department of Pediatrcs, Medical University of Graz. Echocardiography was performed with a Vivid 7 Pro ultrasonic device (10 MHz sector transducer General Electric, Fairfield, CT,
USA) including assessment of any form of severe structural heart disease, volume status, contractility, ejection fraction, superior vena cava (SVC) flow and ductal patency. If blood pressure was at the low side of the normal range (MABP higher but close to gestational age) and/or in echocardiography the attending neonatologist considered volume status/cardiac output to be low and/or SVC flow to be low (routinely $\leq 40 \mathrm{~mL} / \mathrm{kg}$ per minute), the neonatologist had to consider the following interventions: (i) administration of fluid bolus of $10 \mathrm{ml} / \mathrm{kg}$ (normal saline) $(23,24)$, (ii) if ventilated: decrease of mean airway pressure $(23,25,26)$, or (iii) in case of patent ductus arteriosus (PDA): medical treatment for DA closure (23).

\section{DURATION}

Near-infrared spectroscopy monitoring and predefined clinical interventions started within $6 \mathrm{~h}$ after birth and lasted for $24 \mathrm{~h}$ (i.e., 24-30 h after birth). Arterial blood pressure was measured for $48 \mathrm{~h}$ after study inclusion (i.e., $24 \mathrm{~h}$ during and $24 \mathrm{~h}$ after NIRS measurement and predefined interventions). Clinical follow-up was conducted until term age or until discharge, whatever occurred first.

\section{OUTCOME MEASURES}

Demographic, perinatal data, respiratory support, main diagnoses, and infections were documented in each neonate. For the diagnosis of any infection, blood samples were taken from the neonate on the first and second day of life and CRP and leukocyte counts were determined. A blood culture was taken on the first day of life (either infants' blood or cord blood). Infection was defined, when the neonate had clinical signs and a positive blood culture and/or a CRP value above the cutoff value of $10 \mathrm{mg} / \mathrm{l}$.

For analyzes, mean values of arterial oxygen saturation $\left(\mathrm{SpO}_{2}\right)$, $\mathrm{HR}, \mathrm{MABP}, \mathrm{cTOI}$, and pTOI for each hour were calculated in each neonate. Primary outcome was burden of hypotension in $\% \mathrm{mmHg}$ of $\mathrm{MABP}<$ gestational age and/or use of catecholamines during $48 \mathrm{~h}$ after initiation of NIRS measurements.

Secondary outcome measures were incidence of cerebral injury (IVH any grade or PVL any grade) and mortality until term age or until discharge. Cerebral ultrasound was performed at the beginning and end of NIRS measurements, at days 4,7 , and 14 and before discharge.

\section{STATISTICS}

Data are presented as mean and standard deviation (SD) or median and interquartile range (IQR) for continuous data and absolute and relative frequency for categorical data, respectively. Differences between NIRS group and control group were analyzed using $t$-test or Mann-Whitney $U$-test for continuous data and Chi-square test or Fishers's exact test for continuous data. A $p$-value of $<0.05$ was considered statistical significant. Statistical analyses were performed using SPSS 24.0 (SPSS, Chicago, IL, USA). 


\section{RESULTS}

During the study period from October 2013 until December 2016 there were 1,198 deliveries of eligible infants $<37+0$ weeks of gestation. 1,090 neonates (91.0\%) were not enrolled mainly due to the research team not being available or lack of informed consent. In 108 neonates $(9.0 \%)$, NIRS measurements were started. We excluded data of (i) eight neonates because measurements started beyond $6 \mathrm{~h}$ after birth, (ii) one neonate because no NIRS data were recorded, and (iii) one neonate because parental consent was withdrawn during measurements. Thus, 98 preterm infants ( $n=49$ in each group) were enrolled and analyzed (Figure 1). No severe adverse reactions were observed.

In the NIRS group, 20 mothers had premature rupture of membranes with/without infection and 29 mothers had primarily signs of infection/inflammation. In the control group, 22 mothers had premature rupture of membranes with/without infection and 27 mothers primarily signs of infection/inflammation. There were no significant differences in demographic characteristics between the groups (Table 1). Lowest birth weight in the NIRS group was 686 and $880 \mathrm{~g}$ in the control group. In the NIRS group, seven neonates were small for date and in the control group eight neonates. In the NIRS group, 25 neonates were admitted to the neonatal intensive care unit due to idiopathic respiratory distress (IRDS), 14 due to transitory respiratory distress of the newborn (TRDN), and 10 due to prematurity. In the control group, 23 neonates were admitted due to IRDS, 16 due to TRDN, and 10 due to prematurity. In the NIRS group, nine neonates were diagnosed with infection (according to criteria) compared to nine neonates in the control group. In each group, two neonates had culture proven infections. None of the neonates needed cardiocirculatory resuscitation with chest compressions. There were no significant

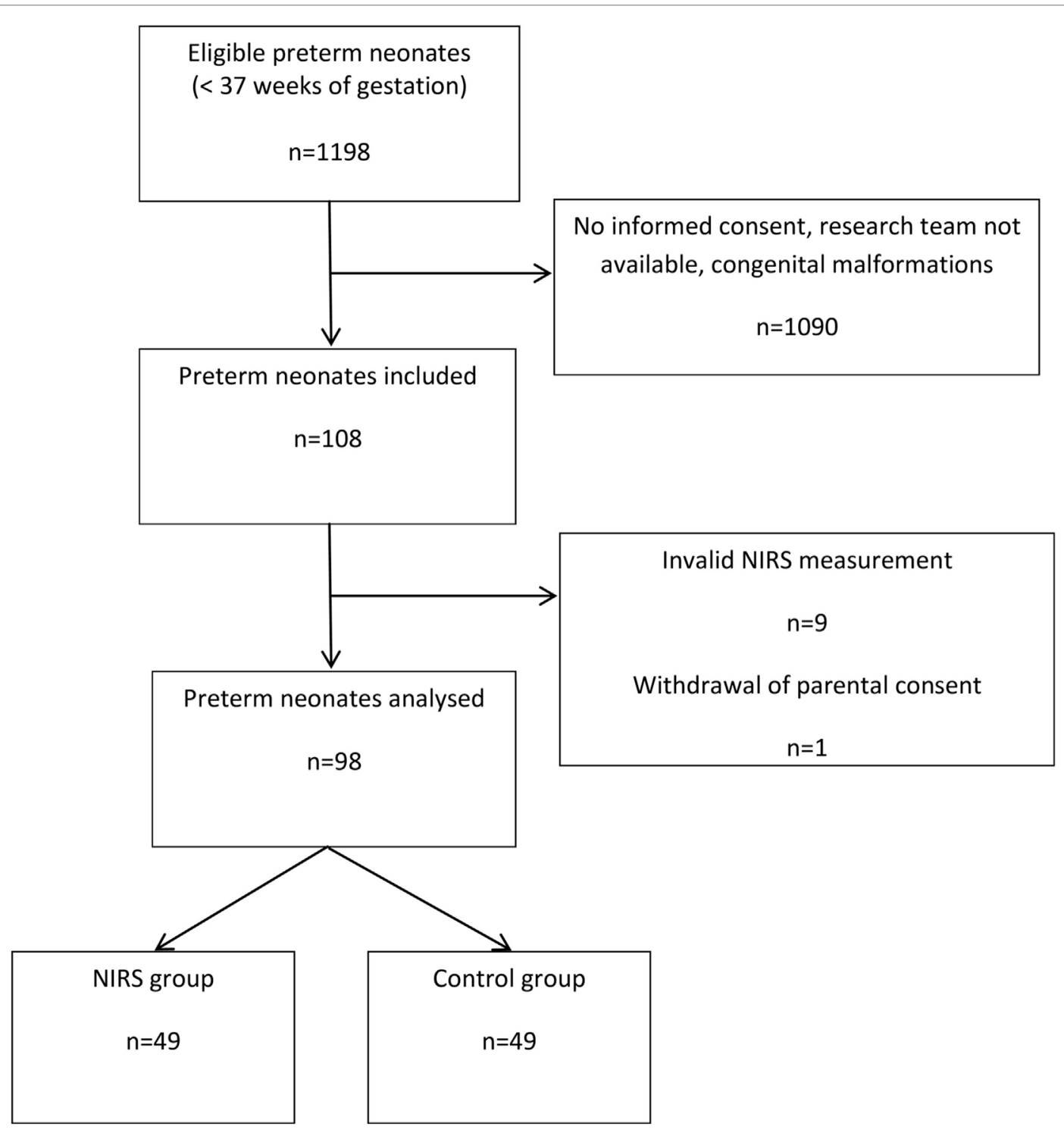

FIGURE 1 | Flow diagram showing the number of included neonates and rationales for exclusion. 
TABLE 1 | Demographic and monitoring data of 49 preterm neonates with NIRS monitoring visible (NIRS group) and of 49 neonates with NIRS monitoring not being visible (control group).

\begin{tabular}{lccc}
\hline & $\begin{array}{c}\text { NIRS group } \\
(\boldsymbol{n}=\mathbf{4 9 )}\end{array}$ & $\begin{array}{c}\text { Control group } \\
(\boldsymbol{n}=\mathbf{4 9 )}\end{array}$ & $\boldsymbol{p}$-Value \\
\hline Gestational age (weeks) & $33.1(32.0-34.0)$ & $33.4(32.3-34.3)$ & 0.354 \\
Birth weight (g) & $1,826 \pm 513$ & $1,845 \pm 504$ & 0.856 \\
Cesarean section, $n$ & 38 & 36 & 0.638 \\
Male, $n$ & 26 & 23 & 0.544 \\
Apgar 5 min & $9(8-9)$ & $9(8-10)$ & 0.696 \\
Umbilical artery pH & $7.30 \pm 0.04$ & $7.29 \pm 0.06$ & 0.177 \\
Respiratory support & 26 & 29 & 0.699 \\
$\quad$ No respiratory support, $n$ & 21 & 17 & \\
Non-invasive respiratory & & & \\
$\quad$ support, $n$ & 2 & 3 & 0.585 \\
Intubated, $n$ & 9 & 7 & 1.000 \\
Surfactant, $n$ \\
Infection, $n$
\end{tabular}

Continuous data are presented as mean \pm SD or median (25th percentile to 75 th percentile) based on distribution.

TABLE 2 | Monitoring parameters and mean arterial blood pressure data of 49 preterm neonates with NIRS monitoring visible (NIRS group) and of 49 neonates with NIRS monitoring not being visible (control group).

\begin{tabular}{lccc}
\hline & $\begin{array}{c}\text { NIRS group } \\
(\boldsymbol{n}=\mathbf{4 9})\end{array}$ & $\begin{array}{c}\text { Control group } \\
(\boldsymbol{n}=\mathbf{4 9})\end{array}$ & $\boldsymbol{p}$-Value \\
\hline $\begin{array}{l}\text { Mean arterial oxygen saturation } \\
\text { first day (\%) }\end{array}$ & $96.5(94.0-98.0)$ & $96.8(95.5-97.6)$ & 0.720 \\
$\begin{array}{l}\text { Mean heart rate first } \\
\text { day (bpm) }\end{array}$ & $144(138-149)$ & $141(137-145)$ & 0.192 \\
$\begin{array}{l}\text { Mean arterial blood pressure } \\
\text { first day (mmHg) }\end{array}$ & $44 \pm 4$ & $43 \pm 5$ & 0.918 \\
$\begin{array}{l}\text { Mean arterial oxygen } \\
\text { saturation second day (\%) }\end{array}$ & $96.9(95.3-98.0)$ & $96.5(94.4-97.5)$ & 0.263 \\
$\begin{array}{l}\text { Mean heart rate second } \\
\text { day (bpm) }\end{array}$ & $144(141-149)$ & $142(136-149)$ & 0.245 \\
$\begin{array}{l}\text { Mean arterial blood pressure } \\
\text { second day (mmHg) }\end{array}$ & $46 \pm 4$ & $46 \pm 5$ & 0.773 \\
\hline
\end{tabular}

Continuous data are presented as mean \pm SD or median (25th percentile to 75th percentile) based on distribution.

differences in monitoring parameters including MABP between the groups (Table 2).

Near-infrared spectroscopy measurements started earlier in the NIRS group $(0.5 \mathrm{~h})$ than in the control group. However, there were no significant differences in median cTOI and pTOI values between NIRS group and control group during the 24-h measurement period.

In the NIRS group, fewer alarms were recorded compared to the control group (Table 3). Seventeen neonates in the NIRS group $(34.7 \%)$ were examined by echocardiography due to NIRS measurements. In two neonates, echocardiography revealed a PDA (diameter $>1.5 \mathrm{~mm} / \mathrm{kg}$ ), which was treated with ibuprofen in both. In four neonates, a bolus of $10 \mathrm{ml}$ normal saline/kg was administered due to echocardiographic low volume status.

In all, $32.6 \%$ of neonates in the NIRS group and $44.9 \%$ in the control group had hypotensive episodes, without significant differences either in number of neonates or in burden of hypotension
TABLE 3 | NIRS measurements and interventions of 49 preterm neonates with NIRS monitoring visible (NIRS group) and of 49 neonates with NIRS monitoring not being visible (control group).

\begin{tabular}{lccc}
\hline & $\begin{array}{c}\text { NIRS group } \\
(\boldsymbol{n}=\mathbf{4 9 )}\end{array}$ & $\begin{array}{c}\text { Control group } \\
(\boldsymbol{n}=\mathbf{4 9 )}\end{array}$ & $\boldsymbol{p}$-Value \\
\hline $\begin{array}{l}\text { Age at initiation, hours } \\
\text { after birth }\end{array}$ & $2.0(1.5-3.5)$ & $2.5(2.0-4.0)$ & $0.039^{*}$ \\
$\begin{array}{l}\text { Mean cerebral tissue } \\
\text { oxygenation index (\%) }\end{array}$ & $70.5(64.8-74.4)$ & $71.2(66.6-74.2)$ & 0.548 \\
$\begin{array}{l}\text { Mean peripheral tissue } \\
\text { oxygenation index (\%) }\end{array}$ & $73.7(69.7-77.8)$ & $72.6(69.8-76.5)$ & 0.354 \\
$\begin{array}{l}\text { Alarms/patient, } n \\
\text { Echocardiographies, } n\end{array}$ & $3(2-4)$ & $4(4-5)$ & $0.008^{*}$ \\
Interventions, $n$ & 17 & - & \\
\hline
\end{tabular}

Continuous data are presented as median (25th percentile to 75 th percentile) based on distribution.

${ }^{*} p<0.05$.

TABLE 4 | Burden of arterial hypotension (\%hours) of 49 preterm neonates with NIRS monitoring visible (NIRS group) and of 49 neonates with NIRS monitoring not being visible (control group).

\begin{tabular}{lccc}
\hline & NIRS group & Control group & $\boldsymbol{p}$-Value \\
\hline $48 \mathrm{~h}$ (during and after NIRS measurements) & & \\
\hline All neonates, $n(\%)$ & $49(100)$ & $49(100)$ & \\
Burden in all patients (\% h) & $0.0(0.0-2.1)$ & $0.4(0.0-3.3)$ & 0.313 \\
\hline $\begin{array}{l}\text { Neonates with hypotensive } \\
\text { episodes, } n \text { (\%) }\end{array}$ & $16(32.6)$ & $22(44.9)$ & 0.214 \\
$\begin{array}{l}\text { Burden in neonates with } \\
\text { hypotensive episodes (\% h) }\end{array}$ & $3.0(2.2-6.1)$ & $4.2(1.7-6.3)$ & 0.804 \\
\hline
\end{tabular}

(Table 4). No severe hypotension with need of catecholamine therapy was observed in both groups.

Regarding cerebral injury, only four neonates had an IVH grade 1 , one in the NIRS group and three in the control group. None of the included neonates died.

\section{DISCUSSION}

In the present study, simultaneous peripheral and cerebral NIRS monitoring followed by predefined interventions led to a non-significant reduction in burden of arterial hypotension in preterm neonates. Nonetheless, in six neonates in the NIRS group echocardiography due to a NIRS monitoring alarm was followed by a medical intervention.

The relevance of arterial hypotension and the indication for treatment is still under discussion. Several observational studies have demonstrated no benefit of antihypotensive therapy (27-29). Contradictory to these findings, an analysis of the EPIPAGE cohort just recently revealed that in extremely premature infants an antihypotensive treatment was associated with improved short-term outcomes (30). Thus, preterm neonates with arterial hypotension-defined as MABP being below gestational age in weeks-receive treatment at many neonatal intensive care units at the moment.

Dempsey et al. (31) reported that hypotensive preterm infants with clinical evidence of good perfusion have as good an outcome 
as normotensive patients, while treated low blood pressure was associated with adverse outcome. Hence, it may be beneficial to treat arterial hypotension especially in association with poor perfusion.

The aim of the present study was to recognize poor perfusion (compromised microcirculation) at an early stage, which then may help identifying neonates in need of cardiocirculatory support before macrocirculation is compromised. However, in the present study mainly late and moderate preterm neonates were included. This was responsible for the fact that most of the neonates were stable from a cardiocirculatory standpoint, which is the main limitation of the present findings. Neonates in both groups and especially in the control group only had borderline hypotension and none of the neonates needed inotropes at all. Therefore, effect of simultaneous peripheral and cerebral NIRS measurements followed by predefined interventions on prevention on severe arterial hypotension cannot be ruled out by the present data.

In the present study, echocardiography was performed by a neonatologist, when indicated in the NIRS group. The evaluation of the cardiovascular status of term and preterm infants by a neonatologist is gaining significant interest to support bedside clinical decision making. However, neonatologist performed echocardiography needs to be trained according recommendations to ensure standardization of training and clinical practice guidelines (32).

pTOI was slightly higher compared to cTOI in the present study. At first, this seems contradictory to recent findings. Grossauer et al. (33) described a ratio of cTOI/pTOI of 1.14. However, in her study term neonates with a mean age of 16 days were measured having similar cTOI with $70.4 \%$ and a much lower pTOI with $62.1 \%$ compared to present values. The different findings may be explained by a physiological decrease of pTOI during the first days after birth. pTOI has been described to decrease from 67 to $61 \%$ from the first to the third day after birth (20-22).

Recently we described that in stable preterm and term neonates with inflammatory processes peripheral oxygenation is lower compared to neonates without inflammatory processes (9). The mean pTOI value of the present study was within the range of recently published pTOI of neonates with and without inflammatory processes. This may be explained by the fact that neonates with and without inflammation/infection/sepsis were distributed equally in both groups.

Four neonates had a mild IVH and none of the included neonates died. These low numbers may be explained by the fact, that mainly moderate and late preterm neonates were included. Rapid, harmful alterations in blood pressure with hypo- or hyperperfusion have not been observed $(34,35)$. In addition, Binder et al. (36) demonstrated that mild short-term hypotensive episodes in preterm infants did not affect cerebral oxygen saturation, suggesting that cerebral autoregulation is maintained in case of borderline hypotension and may protect infants from cerebral injury.

\section{LIMITATIONS}

Our main limitation is that most neonates included were moderate to late preterm neonates in cardiocirculatory stable conditions.
Therefore, none of the neonates in the NIRS group and even none in the control group had severe arterial hypotension or was treated with inotropes. As a further fact of that inclusion none of the neonates died or had severe cerebral injury.

Near-infrared spectroscopy measurements started significantly earlier in the NIRS group compared to the control group. However, the observed difference was only $30 \mathrm{~min}$ and may not be of clinical relevance.

In the control group, the number of recorded potential alarms was higher compared to the NIRS group. Explanation might be that neonates with alarms and low arterial blood pressure in the NIRS group received treatment, causing a reduction in cTOI/ pTOI ratio increase over time.

\section{CONCLUSION}

In the present study, in preterm neonates simultaneous peripheral and cerebral NIRS measurements followed by dedicated interventions led to a non-significant reduction in burden of arterial hypotension. The present study did not prove that this approach resulted in significant better outcome, but that due to the trend these simultaneous peripheral and cerebral NIRS measurements may have the potential to become a clinical tool to recognize disturbances of peripheral microcirculation at an early stage before macrocirculatory impairment becomes evident. The described method should therefore be evaluated in more sick neonates with higher risk of arterial hypotension (e.g., neonates with proven sepsis or neonates in need of mechanical ventilation) and more preterm neonates.

\section{ETHICS STATEMENT}

This study was carried out in accordance with the recommendations of Regional Committees on Biomedical Research Ethics with written informed consent from all subjects. All subjects gave written informed consent in accordance with the Declaration of Helsinki. The protocol was approved by the Ethikkommission, Medizinische Universität Graz, Austria.

\section{AUTHOR CONTRIBUTIONS}

Conception and design: GP, AA, and BU. Collection and assembly of data: GP, NH, NB-S, BS, LM, JS, and JP. Analysis and interpretation of the data: GP, NH, NB-S, BS, LM, JS, AA, JP, and BU. Drafting of the article: GP and AA. Critical revision of the article for important intellectual content: GP, NH, NB-S, BS, LM, JS, AA, JP, and BU. Final approval of the article: GP, NH, NB-S, BS, LM, JS, AA, JP, and BU.

\section{FUNDING}

Supported by funds of the Österreichische Nationalbank (Austrian Central Bank, Anniversary Fund, project number: 15351). The funder had no role in study design, data collection and analysis, decision to publish, or preparation of the manuscript. 


\section{REFERENCES}

1. Barrington KJ, Dempsey EM. Cardiovascular support in the preterm: treatments in search of indications. J Pediatr (2006) 148(3):289-91. doi:10.1016/j. jpeds.2005.12.056

2. Dasgupta SJ, Gill AB. Hypotension in the very low birth weight infant: the old, the new, and the uncertain. Arch Dis Child Fetal Neonatal Ed (2003) Ed 88:F450-4. doi:10.1136/fn.88.6.F450

3. Al-Aweel I, Pursley DM, Rubin LP, Shah B, Weisberger S, Richardson DK. Variations in prevalence of hypotension, hypertension, and vasopressor use in NICUs. J Perinatol (2001) 21:272-8. doi:10.1038/sj.jp.7210563

4. Arvind S. Haemodynamically unstable preterm infant: an unresolved management conundrum. Eur J Pediatr (2011) 170:1237-45. doi:10.1007/ s00431-011-1435-4

5. Watkins AM, West CR, Cooke RW. Blood pressure and cerebral haemorrhage and ischaemia in very low birthweight infants. Early Hum Dev (1989) 19:103-10. doi:10.1016/0378-3782(89)90120-5

6. Bada HS, Korones SB, Perry EH, Arheart KL, Ray JD, Pourcyrous M, et al. Mean arterial blood pressure changes in premature infants and those at risk for intraventricular hemorrhage. J Pediatr (1990) 117:607-14. doi:10.1016/ S0022-3476(05)80700-0

7. Miall-Allen VM, de Vries LS, Whitelaw AG. Mean arterial blood pressure and neonatal cerebral lesions. Arch Dis Child (1987) 62:1068-9. doi:10.1136/ adc.62.10.1068

8. Tsuji M, Saul JP, du Plessis A, Eichenwald E, Sobh J, Crocker R, et al. Cerebral intravascular oxygenation correlates with mean arterial pressure in critically ill premature infants. Pediatrics (2000) 106(4):625-32. doi:10.1542/ peds.106.4.625

9. Pichler G, Pocivalnik M, Riedl R, Pichler-Stachl E, Zotter H, Müller W, et al. $\mathrm{C}$ reactive protein: impact on peripheral tissue oxygenation and perfusion in neonates. Arch Dis Child Fetal Neonatal Ed (2012) 97(6):F444-8. doi:10.1136/ archdischild-2011-300578

10. Parker MM. Pediatric definitions for sepsis: it's about time! Pediatr Crit Care Med (2005) 6:83-4. doi:10.1097/01.PCC.0000149307.02324.05

11. Bateman RM, Sharpe MD, Ellis CG. Bench-to-bedside review: microvascular dysfunction in sepsis-hemodynamics, oxygen transport, and nitric oxide. Crit Care (2003) 7:359-73. doi:10.1186/cc1940

12. Spronk PE, Zandstra DF, Ince C. Bench-to-bedside review: sepsis is a disease of the microcirculation. Crit Care (2004) 8:462-8. doi:10.1186/ cc2894

13. Nanas S, Gerovasili V, Renieris P, Angelopoulos E, Poriazi M, Kritikos K, et al. Non-invasive assessment of the microcirculation in critically ill patients. Anaesth Intensive Care (2009) 37:733-9.

14. Ince C. The microcirculation is the motor of sepsis. Crit Care (2005) 9 (Suppl 4):S13-9. doi:10.1186/cc3135

15. Höller N, Urlesberger B, Mileder L, Baik N, Schwaberger B, Pichler G. Peripheral muscle near-infrared spectroscopy in neonates: ready for clinical use? A systematic qualitative review of the literature. Neonatology (2015) 108(4):233-45. doi:10.1159/000433515

16. Caicedo A, De Smet D, Naulaers G, Ameye L, Vanderhaegen J, Lemmers P, et al. Cerebral tissue oxygenation and regional oxygen saturation can be used to study cerebral autoregulation in prematurely born infants. Pediatr Res (2011) 69(6):548-53. doi:10.1203/PDR.0b013e3182176d85

17. da Costa CS, Greisen G, Austin T. Is near-infrared spectroscopy clinically useful in the preterm infant? Arch Dis Child Fetal Neonatal Ed (2015) 100(6):F558-61. doi:10.1136/archdischild-2014-307919

18. Suresh V, Marson AG, Appleton RE, Beirne M, Weindling AM. Relationship between blood pressure, cerebral electrical activity, cerebral fractional oxygen extraction, and peripheral blood flow in very low birth weight newborn infants. Pediatr Res (2006) 59(2):314-9. doi:10.1203/01.pdr.0000199525. $08615.1 \mathrm{f}$

19. Meybohm P, Renner J, Boening A, Cavus E, Gräsner JT, Grünewald M, et al. Impact of norepinephrine and fluid on cerebral oxygenation in experimental hemorrhagic shock. Pediatr Res (2007) 62(4):440-4. doi:10.1203/ PDR.0b013e3181425858

20. Pichler G, Pocivalnik M, Riedl R, Pichler-Stachl E, Morris N, Zotter H, et al. 'Multi-associations': predisposed to misinterpretation of peripheral tissue oxygenation and circulation in neonates. Physiol Meas (2011) 32(8):1025-34. doi:10.1088/0967-3334/32/8/003
21. Pichler G, Grossauer K, Klaritsch P, Kutschera J, Zotter H, Müller W, et al. Peripheral oxygenation in term neonates. Arch Dis Child Fetal Neonatal Ed (2007) 92(1):F51-2. doi:10.1136/adc.2005.089037

22. Naulaers G, Morren G, Van Huffel S, Casaer P, Devlieger H. Measurement of tissue oxygenation index during the first three days in premature born infants. Adv Exp Med Biol (2003) 510:379-83. doi:10.1007/978-1-4615-0205-0_63

23. Pellicer A, Greisen G, Benders M, Claris O, Dempsey E, Fumagally M, et al. The SafeBoosC phase II randomised clinical trial: a treatment guideline for targeted near-infrared-derived cerebral tissue oxygenation versus neonatology standard treatment in extremely preterm infants. Neonatology (2013) 104:171-8. doi:10.1159/000351346

24. Osborn D, Evans N, Kluckow M. Randomized trial of dobutamine versus dopamine in preterm infants with low systemic blood flow. J Pediatr (2002) 140(2):183-91. doi:10.1067/mpd.2002.120834

25. Evans N, Kluckow M. Early determinants of right and left ventricular output in ventilated preterm infants. Arch Dis Child Fetal Neonatal Ed (1996) 74(2):F88-94. doi:10.1136/fn.74.2.F88

26. Kluckow M, Evans N. Relationship between blood pressure and cardiac output in preterm infants requiring mechanical ventilation. J Pediatr (1996) 129(4):506-12. doi:10.1016/S0022-3476(96)70114-2

27. Batton B, Zhu X, Fanaroff J, Kirchner HL, Berlin S, Wilson-Costello D, et al. Blood pressure, anti-hypotensive therapy, and neurodevelopment in extremely preterm infants. J Pediatr (2009) 154:351-7, 357.e1. doi:10.1016/j. jpeds.2008.09.017

28. Batton B, Li L, Newman NS, Das A, Watterberg KL, Yoder BA, et al. Use of antihypotensive therapies in extremely preterm infants. Pediatrics (2013) 131:e1865-73. doi:10.1542/peds.2012-2779

29. Dempsey EM, Al Hazzani F, Barrington KJ. Permissive hypotension in the extremely low birthweight infant with signs of good perfusion. Arch Dis Child Fetal Neonatal Ed (2009) 94:F241-4. doi:10.1136/adc.2007.124263

30. Durrmeyer X, Marchand-Martin L, Porcher R, Gascoin G, Roze JC, Storme L, et al. Abstention or intervention for isolated hypotension in the first 3 days of life in extremely preterm infants: association with short-term outcomes in the EPIPAGE 2 cohort study. Arch Dis Child Fetal Neonatal Ed (2017) 102:490-6. doi:10.1136/archdischild-2016-312104

31. Dempsey EM, Barrington KJ, Marlow N, O’Donnell CP, Miletin J, Naulaers G, et al. Management of hypotension in preterm infants (The HIP Trial): a randomised controlled trial of hypotension management in extremely low gestationalagenewborns. Neonatology (2014) 105(4):275-81.doi:10.1159/000357553

32. de Boode WP, Singh Y, Gupta S, Austin T, Bohlin K, Dempsey E, et al. Recommendations for neonatologist performed echocardiography in Europe: consensus statement endorsed by European Society for Paediatric Research (ESPR) and European Society for Neonatology (ESN). Pediatr Res (2016) 80(4):465-71. doi:10.1038/pr.2016.126

33. Grossauer K, Pichler G, Schmölzer G, Zotter H, Müller W, Urlesberger B. Comparison of peripheral and cerebral tissue oxygenation index in neonates. Arch Dis Child Fetal Neonatal Ed (2009) 94(2):F156. doi:10.1136/ adc. 2008.146654

34. Greisen G. Cerebral blood flow and energy metabolism in the newborn. Clin Perinatol (1997) 24:531-46.

35. Kluckow M, Evans N. Low superior vena cava flow and intraventricular haemorrhage in preterm infants. Arch Dis Child Fetal Neonatal Ed (2000) 82:F188-94. doi:10.1136/fn.82.3.F188

36. Binder-Heschl C, Urlesberger B, Schwaberger B, Koestenberger M, Pichler G. Borderline hypotension: how does it influence cerebral regional tissue oxygenation in preterm infants? J Matern Fetal Neonatal Med (2016) 29(14):2341-6. doi: $10.3109 / 14767058.2015 .1085020$

Conflict of Interest Statement: The authors declare that the research was conducted in the absence of any commercial or financial relationships that could be construed as a potential conflict of interest.

Copyright (C) 2018 Pichler, Höller, Baik-Schneditz, Schwaberger, Mileder, Stadler, Avian, Pansy and Urlesberger. This is an open-access article distributed under the terms of the Creative Commons Attribution License (CC BY). The use, distribution or reproduction in other forums is permitted, provided the original author(s) and the copyright owner are credited and that the original publication in this journal is cited, in accordance with accepted academic practice. No use, distribution or reproduction is permitted which does not comply with these terms. 